\title{
Improved stability of black silicon detectors using aluminum oxide surface passivation
}

Juha Heinonen, Antti Haarahiltunen, Heikki Kettunen, Jukka Jaatinen, Mikko Rossi, et al.

Juha Heinonen, Antti Haarahiltunen, Heikki Kettunen, Jukka Jaatinen, Mikko Rossi, Jouni Heino, Hele Savin, Mikko A. Juntunen, "Improved stability of black silicon detectors using aluminum oxide surface passivation," Proc. SPIE 11852, International Conference on Space Optics - ICSO 2020, 118520T (11 June 2021); doi: 10.1117/12.2599177

SPIE Event: International Conference on Space Optics - ICSO 2021, 2021, Online Only 


\section{International Conference on Space Optics-ICSO 2020}

Virtual Conference

30 March-2 April 2021

Edited by Bruno Cugny, Zoran Sodnik, and Nikos Karafolas
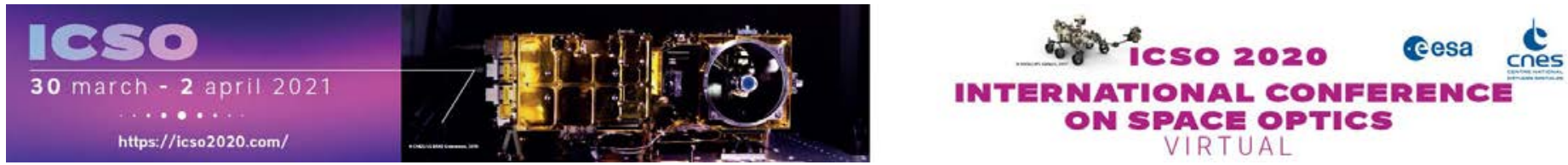

\section{Improved stability of black silicon detectors using aluminum oxide surface passivation}

\section{Cesa isoporeseatings denes}




\title{
Improved stability of black silicon photodiodes using aluminum oxide surface passivation
}

\author{
Juha Heinonen ${ }^{\mathrm{a}}$, Antti Haarahiltunen ${ }^{\mathrm{a}}$, Heikki Kettunen ${ }^{\mathrm{b}}$, Jukka Jaatinen ${ }^{\mathrm{b}}$, Mikko Rossi ${ }^{\mathrm{b}}$, Jouni \\ Heino $^{\mathrm{c}}$, Hele Savin ${ }^{\mathrm{d}}$ and Mikko A. Juntunen ${ }^{\mathrm{a}}$. \\ ${ }^{a}$ ElFys, Inc., Tekniikantie 12, 02150 Espoo, Finland; ${ }^{b}$ Department of Physics, University of \\ Jyväskylä, P.O. Box 35 (YFL), FI-40014 Jyväskylä, Finland; ${ }^{c}$ Detector Laboratory, Helsinki Institute \\ of Physics, P.O.Box 64, 00014 University of Helsinki; ${ }^{\mathrm{d}}$ Department of Electronics and \\ Nanoengineering, Aalto University, Tietotie 3,02150 Espoo, Finland;
}

\begin{abstract}
We have studied how high-energy electron irradiation $(12 \mathrm{MeV}$, total dose $66 \mathrm{krad}(\mathrm{Si}))$ and long term humidity exposure $\left(75 \%, 75^{\circ} \mathrm{C}, 500\right.$ hours) influence the induced junction black silicon or planar photodiode characteristics. In our case, the induced junction is formed using n-type silicon and atomic-layer deposited aluminum oxide $\left(\mathrm{Al}_{2} \mathrm{O}_{3}\right)$, which contains a large negative fixed charge. We compare the results with corresponding planar pn-junction detectors passivated with either with silicon dioxide $\left(\mathrm{SiO}_{2}\right)$ or $\mathrm{Al}_{2} \mathrm{O}_{3}$. The results show that the induced junction detectors remain stable as their responsivity remains nearly unaffected during the electron beam irradiation. On the other hand, the $\mathrm{SiO} 2$ passivated counterparts that included conventional pn-junction degrade heavily, which is seen as strongly reduced UV response. Similarly, after humidity test the response of the induced junction detector remains unaffected, while the pn-junction detectors passivated with $\mathrm{SiO}_{2}$ degrade significantly, for instance, the response at $200 \mathrm{~nm}$ reduces to $50 \%$ from the original value. Interestingly, the pn-junction detectors passivated with $\mathrm{Al}_{2} \mathrm{O}_{3}$ exhibit no degradation of UV response, indicating that the surface passivation properties of $\mathrm{Al}_{2} \mathrm{O}_{3}$ are more stable than $\mathrm{SiO}_{2}$ under the studied conditions. This phenomenon is further confirmed with PC1D simulations suggesting that the UV degradation results from increased surface recombination velocity. To conclude, the results presented here suggest that black silicon photodiodes containing $\mathrm{Al}_{2} \mathrm{O}_{3}$-based induced junction are highly promising alternatives for applications that require the best performance and long-term stability under ionizing and humid conditions.
\end{abstract}

Keywords: black silicon, photodiode, irradiation, humidity

\section{INTRODUCTION}

Silicon is still the dominating material for photo- and radiation detectors, as well as for indirect detection of high energy particles or X-rays. However, it is well known that the response of silicon photodetector is limited ultimately by losses due to reflection, surface recombination and bulk recombination. Recently several solutions have been proposed to overcome these losses. For instance, nanostructured silicon surface, i.e. black silicon, eliminates virtually all reflections due to gradient refractive index[1]. Secondly, Auger recombination is a major bulk loss mechanism in doped pn-junction, but it can be avoided by inducing the junction with a fixed charge incorporated in a dielectric film. Consequently, the combination of black silicon and induced junction can be used to make a photodetector with nearly ideal response [2], [3].

In many applications, the photodetector needs to operate reliably in a hostile environment that could include e.g. high relative humidity or significant doses of radiation. These issues can be averted to some degree with protective packaging but that often also dampens the signal of interest. Therefore, it would be beneficial if the detector could survive the conditions with minimal protection. The study of radiation hardness properties and environmental stability of induced junction black silicon photodiodes were targets of the project "Application of black silicon surface treatment to photodiodes 
and silicon drift detectors" supported by the European Space Agency (ESA). We have recently reported radiation hardness results of conventional and induced junction black silicon PIN photodiodes in electron and proton beam irradiation[4], which indicated that induced junction photodiodes are more stable in case of electron beam irradiation. We present here further results from high-energy electron irradiation $(12 \mathrm{MeV}$, total dose $67 \mathrm{krad}(\mathrm{Si}))$ and long term humidity exposure $\left(75 \%, 75^{\circ} \mathrm{C}, 500\right.$ hours). We compare the stability of responsivity, when junction type and surface passivation layer are varied.

\section{EXPERIMENTAL}

\subsection{1 Sample structures}

The focus of the paper is on (surface passivation) stability of various kinds of photodiode junctions, namely silicon dioxide $\left(\mathrm{SiO}_{2}\right)$ and aluminum oxide $\left(\mathrm{Al}_{2} \mathrm{O}_{3}\right)$ passivated conventional boron implanted pn-junction, and both planar and black silicon induced junctions made by $\mathrm{Al}_{2} \mathrm{O}_{3}$. We present here a collection of the results from PIN and silicon drift detectors (SDD) processed during the ESA-project. The different types of PIN photodiodes were fabricated on the same n-type silicon substrate wafer $\left(3-10 \mathrm{k} \Omega \mathrm{cm}, 500 \mu \mathrm{m}\right.$-thick). They contained $\mathrm{SiO}_{2}$ passivated pn-junction and both types of induced junction made by $\mathrm{Al}_{2} \mathrm{O}_{3}$ [4] excluding aluminum oxide passivated pn-junction. The active area of PIN photodiodes was $7.9 \mathrm{~mm}^{2}$. SDDs with $\mathrm{SiO}_{2}$ passivated pn-junction and active area of $29 \mathrm{~mm}^{2}$ were also processed on similar substrates. During the project, SDDs were also processed on slightly different n-type wafer ( $>10 \mathrm{k} \Omega \mathrm{cm}, 675 \mu \mathrm{m}$-thick), in which the front junction was realized using $\mathrm{Al}_{2} \mathrm{O}_{3}$ passivated pn-junction and both types of induced junctions made by $\mathrm{Al}_{2} \mathrm{O}_{3}$. The active area of SDDs was $19.6 \mathrm{~mm}^{2} . \mathrm{SiO}_{2}$ and $\mathrm{Al}_{2} \mathrm{O}_{3}$ layer thicknesses were always $80 \mathrm{~nm}$ and $20 \mathrm{~nm}$, respectively. These thicknesses were measured from the process control samples instead of finished detectors. Inductively coupled plasma reactive ion etching (ICP-RIE) was used to etch black silicon at cryogenic temperature and atomic layer deposition (ALD) was used to deposit $\mathrm{Al}_{2} \mathrm{O}_{3}$. To summarize, SDD's entrance window and PIN photodiode junctions with different passivation layers comply the intended combinations as shown in Table I.

Table I Collection of structures used in this study. $\mathrm{SiO}_{2}$ thickness was $80 \mathrm{~nm}$ and $\mathrm{Al}_{2} \mathrm{O}_{3}$ thickness was $20 \mathrm{~nm}$.

\begin{tabular}{|l|l|l|l|}
\hline Detector structure & Junction & Passivation layer & n-type substrate \\
\hline SDD & Boron implanted pn-junction & $\mathrm{Al}_{2} \mathrm{O}_{3}$ & $>10 \mathrm{k} \Omega \mathrm{cm}, 675 \mu \mathrm{m}$-thick \\
\hline SDD & Planar or black silicon induced junction & $\mathrm{Al}_{2} \mathrm{O}_{3}$ & $>10 \mathrm{k} \Omega \mathrm{cm}, 675 \mu \mathrm{m}$-thick \\
\hline SDD & Boron implanted pn-junction* & $\mathrm{SiO}_{2}$ & $3-10 \mathrm{k} \Omega \mathrm{cm}, 500 \mu \mathrm{m}$-thick \\
\hline PIN & Boron implanted pn-junction* & $\mathrm{SiO}_{2}$ & $3-10 \mathrm{k} \Omega \mathrm{cm}, 500 \mu \mathrm{m}$-thick \\
\hline
\end{tabular}

*Same boron doping profile

\subsection{Environmental stress and electron beam irradiation}

The humidity test was performed at the Helsinki Institute of Physics (HIP), in an ESPEC ENX12-7.5 environmental chamber. The detector chips were placed in the chamber without any packaging, on a 3D-printed holder designed for the tests. Both PIN and SDD devices were tested together. The holders were placed on top of a clean aluminum plate and inserted in the center of the chamber at room temperature. The test conditions in the chamber were 500 hours at $70{ }^{\circ} \mathrm{C}$ and $70 \%$ relative humidity. First, the chamber temperature was raised to $70{ }^{\circ} \mathrm{C}$, and after that the humidity was raised to $70 \%$, during a total ramping time of approximately $15 \mathrm{~min}$.

The electron irradiation tests were performed at the Accelerator Laboratory at the University of Jyväskylä, Finland, using the RADiation Effects Facility (RADEF). The electron beam was generated using recommissioned linear electron accelerator (LINAC) Varian Clinac ${ }^{\circledR}$ medical accelerator. The dose rate was the maximum of LINAC, i.e. $0.76 \mathrm{krad} / \mathrm{min}$ (in silicon), which means that the electron beam consists of a series of $5 \mu$ s pulses with a period of $5 \mathrm{~ms}$ (which corresponds 
to a duty cycle of $0.1 \%)$. The total dose of electron beam radiation was $66.9 \mathrm{krad}(\mathrm{Si})$. The front junction of SDD and PIN photodiodes were under $100 \mathrm{~V}$ reverse bias during electron beam irradiation.

\subsection{3 External quantum efficiency measurements and simulations}

External Quantum Efficiency (EQE) spectra were measured with a setup where the photodiode was illuminated with Bentham ILD-D2-QH-24 dual-lamp light source. The lamp was coupled to Bentham TMC300_0060 monochromator where the wavelength was selected with $10 \mathrm{~nm}$ bandwidth and was then focused on the photodiode. The EQE values were calibrated against Newport 818-UV photodetector. In order to identify and quantify the damage mechanisms, we used the PC-1D simulator version 5.9 [5] to calculate the internal quantum efficiency (IQE) of the photodetectors as described in Refs. [4], [6]. The front surface reflection $R_{f}$ was calculated using the free online calculator available by Filmtronics [7] and EQE can be obtained from the simulated IQE with:

$$
E Q E=\left(1-R_{f}\right) I Q E
$$

The simulations used the default Auger recombination and radiative recombination parameters of the simulation tool. For the initial EQE, long (several ms) Shockley-Read-Hall bulk recombination lifetime was used. Additionally, a relatively low $100 \mathrm{~cm} / \mathrm{s}$ effective back surface recombination velocity $S_{\text {eff }}$ was used together with the mirroring effect of the back surface to get reasonable fitting of the initial EQE. Furthermore, the front surface $S_{\text {eff }}$ was set to $10 \mathrm{~cm} / \mathrm{s}$ for $\mathrm{SiO}_{2}$. The $\mathrm{Al}_{2} \mathrm{O}_{3}$ passivation layer and induced junction was modeled with negative surface charge $-2 \times 10^{12} \mathrm{~cm}^{-2}$ and with surface recombination velocity of $2.5 \times 10^{5} \mathrm{~cm} / \mathrm{s}$ for both electrons and holes. The radiation damage and degradation due to humidity were modeled by decreasing the bulk lifetime and/or increasing the front surface recombination. 


\section{RESULTS AND DISCUSSION}

\subsection{1 Electron beam irradiation}

a)

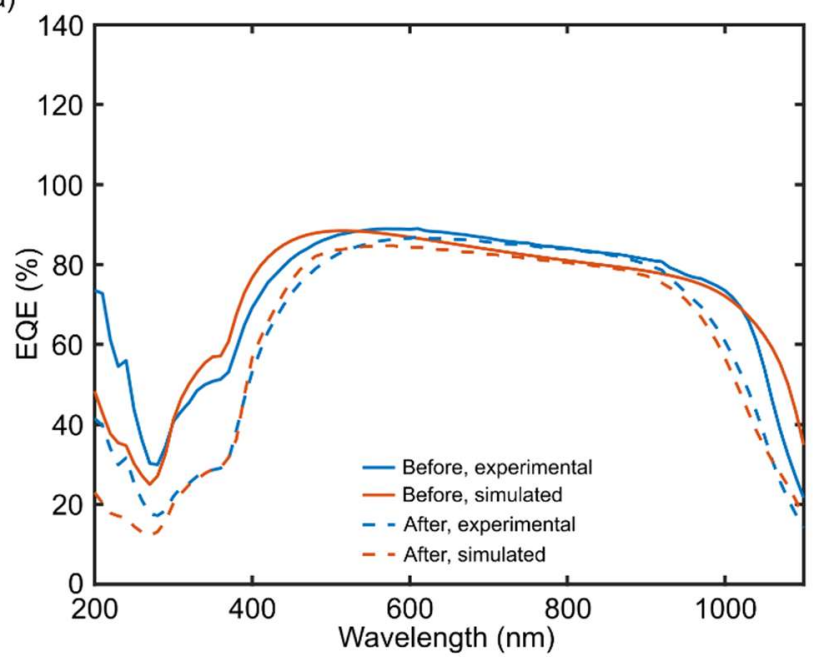

c)

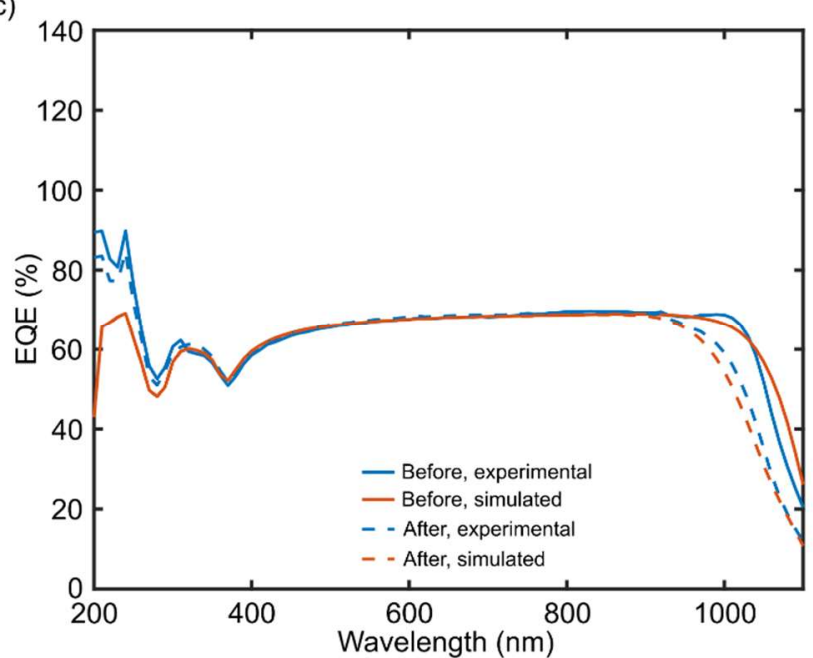

b)

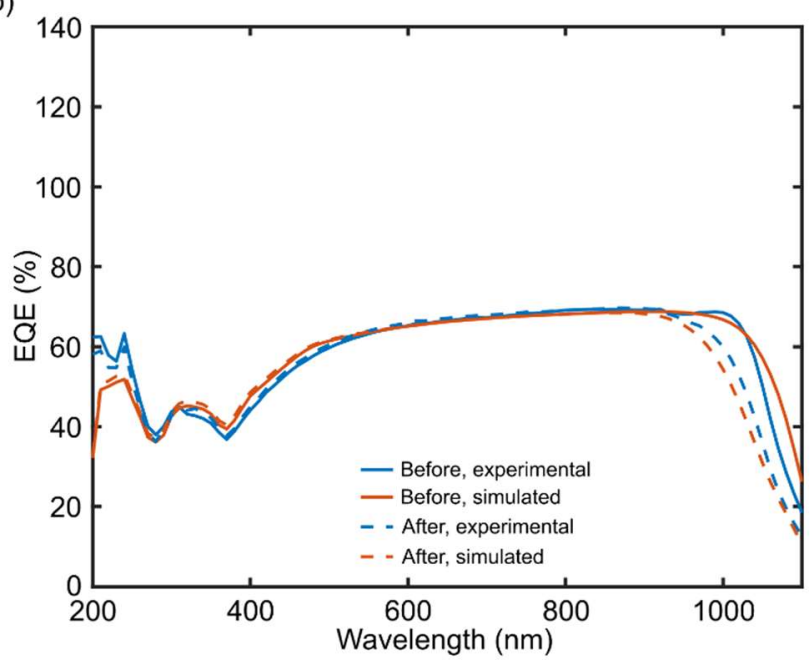

d)

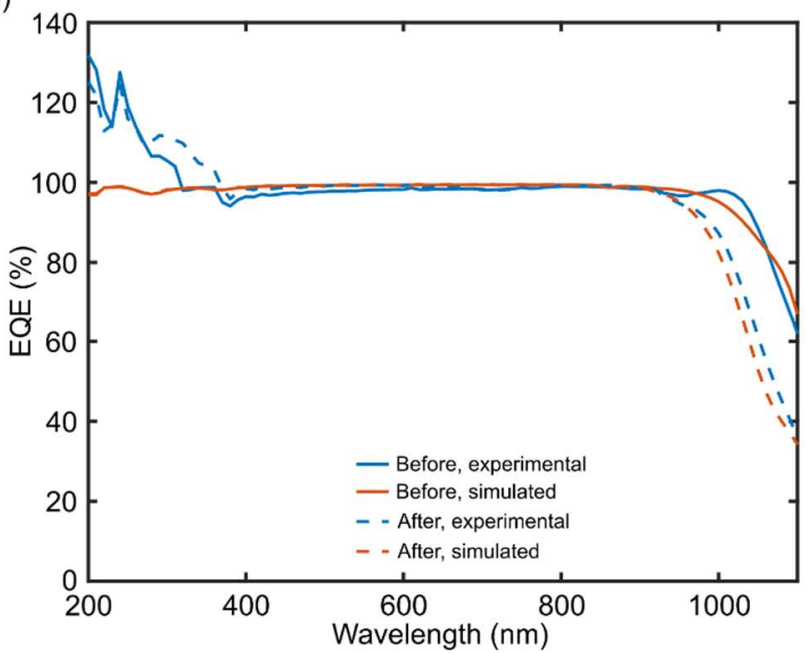

Figure 1. Measured (blue) and simulated (red) spectral response of SDDs having a) boron implanted pn-junction with $\mathrm{SiO}_{2}$ passivation b) boron implanted pn-junction with $\mathrm{Al}_{2} \mathrm{O}_{3}$ passivation c) planar $\mathrm{Al}_{2} \mathrm{O}_{3}$ induced junction and d) $\mathrm{Al}_{2} \mathrm{O}_{3}$ induced junction black silicon structures before (solid lines) and after (dashed lines) electron irradiation with $100 \mathrm{~V}$ reverse bias applied during irradiation.

Figure 1 shows the simulated and measured EQE curves of different types of SDDs before and after the $66.9 \mathrm{krad}(\mathrm{Si})$ electron beam irradiation. For all devices, the EQE decreases at long wavelengths $>900 \mathrm{~nm}$. This change can be simulated by decreasing the silicon bulk lifetime to $20-40 \mu$ s wich models bulk damage generation during the irradiation. In addition, the ultraviolet response decreases in $\mathrm{SiO}_{2}$ passivated boron implanted SDDs when irradiated with electron beam under 100 $\mathrm{V}$ reverse bias (Figure 1a). This change is similar than what has been reported earlier for $\mathrm{SiO}_{2}$ passivated boron implanted PINs [4]. In case of SDDs, the impact of electron beam irradiation on EQE can be explained by using $\mathrm{S}_{\mathrm{eff}}$ of $5.5 \times 10^{5} \mathrm{~cm} / \mathrm{s}$ at the $\mathrm{Si}_{-} \mathrm{SiO}_{2}$ interface (Figure 1a), although this value is lower than $1 \times 10^{7} \mathrm{~cm} / \mathrm{s}$ which was concluded in the previous experiments [4]. This might indicate that the exact degradation rate is dependent on the processing history of the detector. However, when induced junction (Figure 1c,1d) or $\mathrm{Al}_{2} \mathrm{O}_{3}$ passivation (Figure 1b) is used, the EQE of UV region is stable indicating that $\mathrm{Al}_{2} \mathrm{O}_{3}$ passivation has better radiation hardness than $\mathrm{SiO}_{2}$. 


\subsection{2 Humidity stress}

a)

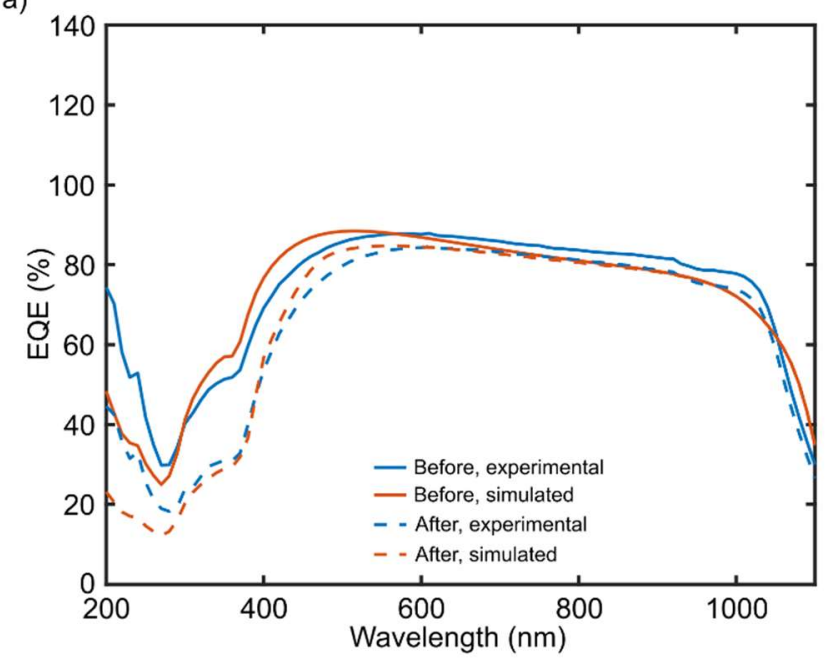

c)

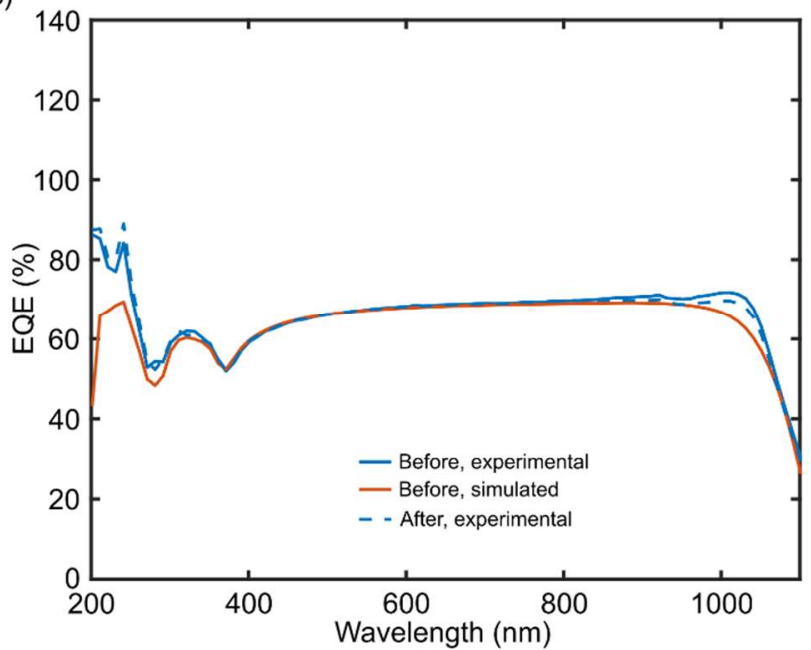

b)

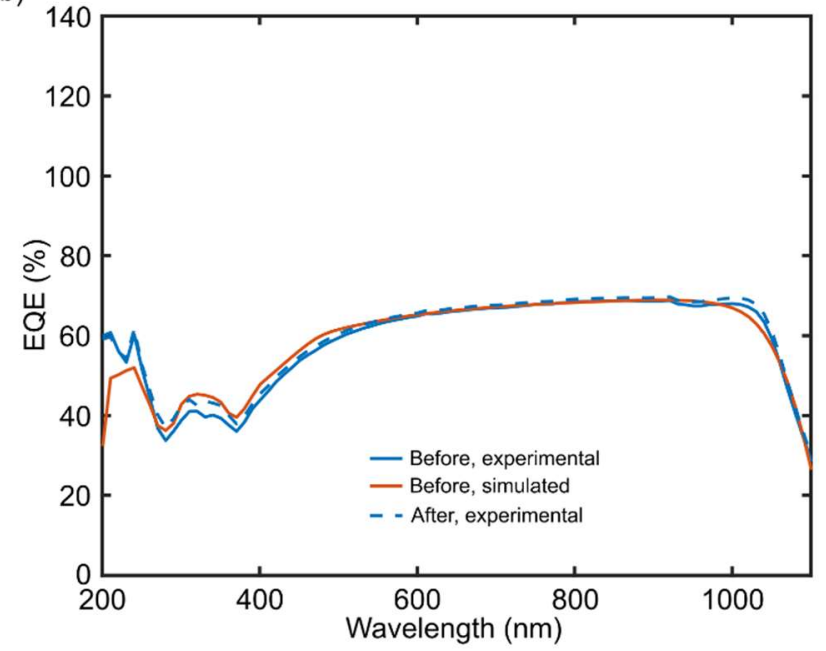

d)

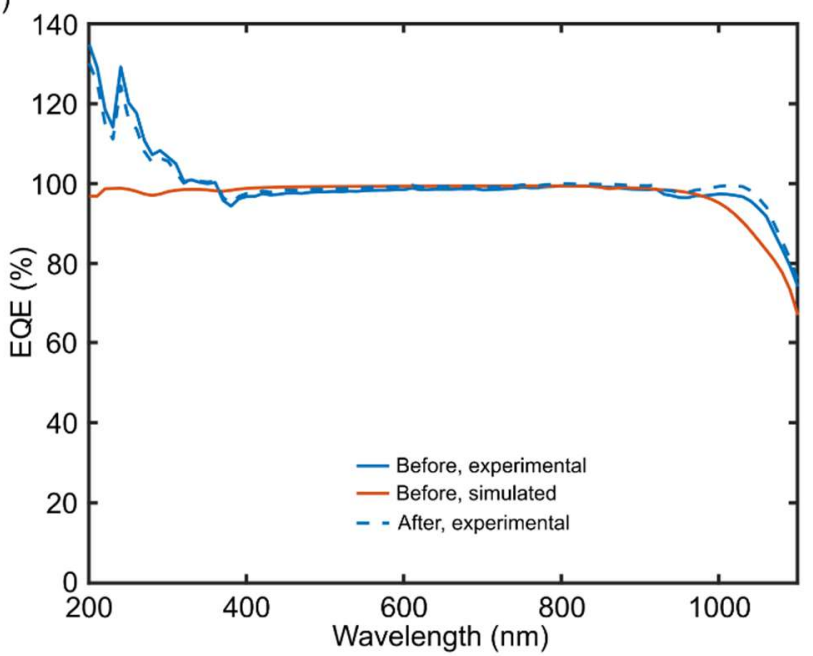

Figure 2. Measured (blue) and simulated (red) spectral response of SDD having a) boron implanted pn-junction with $\mathrm{SiO}_{2}$ passivation b) boron implanted pn-junction with $\mathrm{Al}_{2} \mathrm{O}_{3}$ passivation c) planar $\mathrm{Al}_{2} \mathrm{O}_{3}$ induced junction and d) $\mathrm{Al}_{2} \mathrm{O}_{3}$ induced junction black silicon structures before (solid lines) and after (dashed lines) treatment in environmental chamber.

Figure 2 shows the simulated and measured EQE curves of different types of SDDs before and after the $500 \mathrm{~h}$ humidity stress test. Different device types are showing clearly different EQE behavior. The EQE of boron implanted $\mathrm{SiO}_{2}$ passivated junction degraded clearly after the humidity stress as can be seen from Figure 2 a). The decrease in EQE after the test can be simulated assuming surface recombination velocity of $5.5 \times 10^{5} \mathrm{~cm} / \mathrm{s}$ at the $\mathrm{Si}_{-} \mathrm{SiO}_{2}$ interface. Once again, when induced junction (Figure 2c,2d) or $\mathrm{Al}_{2} \mathrm{O}_{3}$ passivation (Figure 2b) is used, the EQE of UV region is stable. The results from boron implanted $\mathrm{SiO}_{2}$ passivated PIN detector before and after humidity stress are shown in Figure 3. Similar degradation is seen than in $\mathrm{SiO}_{2}$ passivated SDD. With long wavelengths, no degradation is seen with any detector type.

The $\mathrm{Al}_{2} \mathrm{O}_{3}$ film has been used as a moisture diffusion barrier [8]. It has also been reported that humidity treatment at the doped surfaces in case of $\mathrm{SiO}_{2}$ passivation leads to reduction of chemical surface passivation [9] while the $\mathrm{Al}_{2} \mathrm{O}_{3}$ surface passivation seems to be more stable [9]-[11] at least as long as no water condensates on the surface. Our results are in line with these reports suggesting that water vapor diffusion through the thicker $\mathrm{SiO}_{2}$ (assuming that the oxidation reaction at the interface is not limiting) is faster than through the $20 \mathrm{~nm}$ thick $\mathrm{Al}_{2} \mathrm{O}_{3}$ layer. 


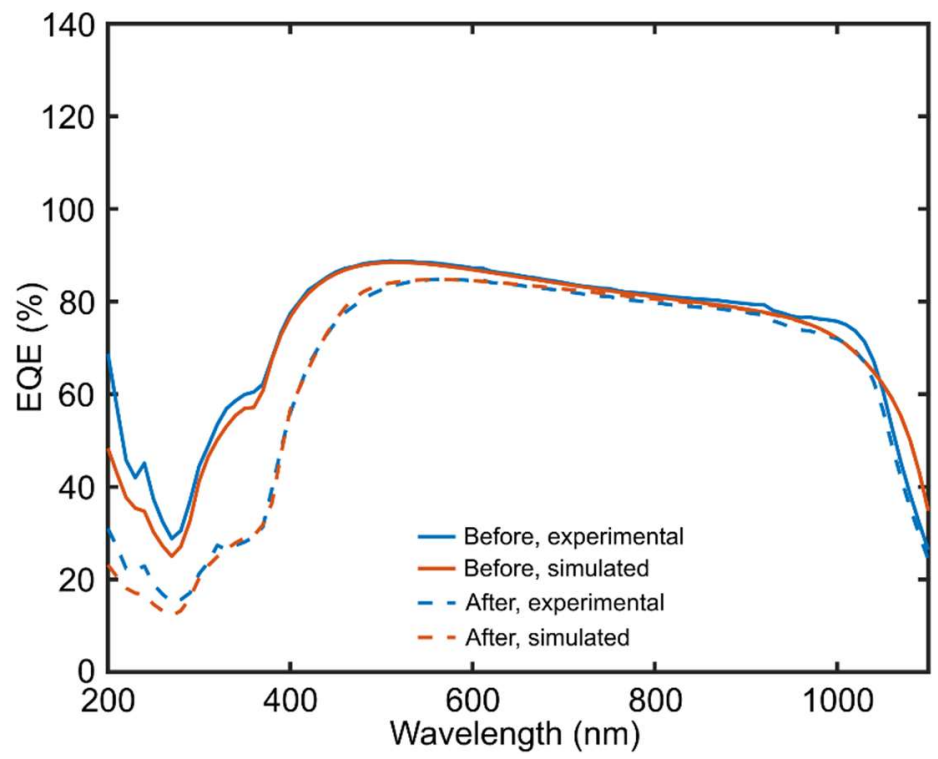

Figure 3. Measured (blue) and simulated (red) spectral response of boron implanted $\mathrm{SiO}_{2}$ passivated PIN before (solid lines) and after (dashed lines) the treatment in environmental chamber.

\section{CONCLUSIONS}

The EQE measurements before and after humidity stress showed that induced junction or pn-junction passivated with $\mathrm{Al}_{2} \mathrm{O}_{3}$ remains unaffected whereas the response of pn-junction passivated with $\mathrm{SiO}_{2}$ was severely degraded at ultraviolet wavelengths. We can conclude that $\mathrm{Al}_{2} \mathrm{O}_{3}$ (induced junction or surface passivation) is more stable in environment containing water vapour. Similar observation was made during electron beam irradiation, in which the response of $\mathrm{SiO}_{2}$ passivated pn-junction devices was almost completely lost at ultraviolet wavelengths while the response of $\mathrm{Al}_{2} \mathrm{O}_{3}$ (induced junction or surface passivation) devices was not affected. These results suggest that black silicon detectors containing $\mathrm{Al}_{2} \mathrm{O}_{3}$-based induced junction are highly promising alternatives for applications that require high sensitivity together with long-term stability under ionizing and/or humid conditions.

\section{ACKNOWLEDGEMENTS}

This work was supported by the ESA General Support Technology Programme (GSTP) [Contract No. 4000124043/18/NL/AR, Application of black silicon surface treatment to photodiodes and silicon drift detectors] and the ESA Basic Technology Research Programme (TRP) Contract Nr. [4000124504/18/NL/AR]. The Accelerator Laboratory at the University of Jyväskylä, Finland is acknowledged for the use of the RADiation Effects Facility (RADEF) for this work. Ametek Finland is acknowledged for help in detector processing. We acknowledge the provision of facilities by Aalto University at OtaNano - Micronova Nanofabrication Centre and Helsinki Institute of Physics. 


\section{REFERENCES}

[1] A. A. Elsayed, Y. M. Sabry, F. Marty, T. Bourouina, and D. Khalil, "Optical modeling of black silicon using an effective medium/multi-layer approach," Opt. Express, vol. 26, no. 10, p. 13443, May 2018, doi:

$10.1364 /$ oe.26.013443.

[2] M. A. Juntunen, J. Heinonen, V. Vähänissi, P. Repo, D. Valluru, and H. Savin, "Near-unity quantum efficiency of broadband black silicon photodiodes with an induced junction," Nat. Photonics, vol. 10, no. 12, pp. 777-781, 2016, doi: 10.1038/nphoton.2016.226.

[3] M. Garin et al., "Black-Silicon Ultraviolet Photodiodes Achieve External Quantum Efficiency above 130\%," Phys. Rev. Lett., vol. 125, no. 11, p. 117702, Sep. 2020, doi: 10.1103/PHYSREVLETT.125.117702.

[4] J. Heinonen et al., "Results on radiation hardness of black silicon induced junction photodetectors from proton and electron radiation," Nucl. Instruments Methods Phys. Res. Sect. A Accel. Spectrometers, Detect. Assoc. Equip., vol. 977, p. 164294, Oct. 2020, doi: 10.1016/j.nima.2020.164294.

[5] D. A. Clugston and P. A. Basore, "PC1D version 5: 32-bit solar cell modeling on personal computers," in Conference Record of the Twenty Sixth IEEE Photovoltaic Specialists Conference - 1997, 1997, pp. 207-210.

[6] J. Heinonen, T. P. Pasanen, V. Vahanissi, M. A. Juntunen, and H. Savin, "Modeling Field Effect in Black Silicon and Its Impact on Device Performance," IEEE Trans. Electron Devices, vol. 67, no. 4, pp. 1645-1652, Apr. 2020, doi: 10.1109/TED.2020.2975145.

[7] "Spectral Reflectance Calculator for Thin-Film Stacks." https://www.filmetrics.com/reflectance-calculator (accessed Aug. 22, 2019).

[8] T. Hirvikorpi, M. Vähä-Nissi, J. Nikkola, A. Harlin, and M. Karppinen, "Thin A12O3 barrier coatings onto temperature-sensitive packaging materials by atomic layer deposition," Surf. Coatings Technol., vol. 205, no. 21-22, pp. 5088-5092, Aug. 2011, doi: 10.1016/j.surfcoat.2011.05.017.

[9] J. C. JBellet and K. R. McIntosh, "HUMIDITY DEGRADATION OF OXIDE-PASSIVATED DIFFUSED SILICON," in 22nd European Photovoltaic Solar Energy Conference, 2007, pp. 1318-1321, Accessed: Dec. 16, 2019. [Online]. Available:

https://www.researchgate.net/publication/237113951_HUMIDITY_DEGRADATION_OF_OXIDEPASSIVATED_DIFFUSED_SILICON.

[10] I. T. S. Heikkinen, G. Koutsourakis, S. Wood, V. Vähänissi, F. A. Castro, and H. Savin, "Stability of the surface passivation properties of atomic layer deposited aluminum oxide in damp heat conditions," in AIP Conference Proceedings, Aug. 2019, vol. 2147, no. 1, p. 050003, doi: 10.1063/1.5123852.

[11] W. Liang, D. Suh, J. Yu, J. Bullock, and K. J. Weber, "Degradation of the surface passivation of plasma-assisted $\mathrm{ALD} \mathrm{Al}_{2} \mathrm{O}_{3}$ under damp-heat exposure," Phys. status solidi, vol. 212, no. 2, pp. 274-281, Feb. 2015, doi: $10.1002 /$ pssa.201431256. 